\title{
Hemihipertrofia Congénita
}

\author{
Dr. Sergio Zúñiga R.; Dr. Alejandro Zavala B.; Dr. Carlos Pérez L. 1-2
}

\section{Congenital Hemiliypertrophy}

\begin{abstract}
Congenital hemihypertrophy is a rare condition of uncertain origin first described by Meckel in 1822 . It is characterized by asymmetry with enlargement of an entire side or specific regions and uccasionally diffetent anatomic areas on both sides of the body. lis association with various congenital defects (hypospadias, eryptorchidism, renal ectopy, nephromepaly, genital hypertrophy, tnedullary sponge kidney) and increased susceptibility to neoplasm of the kidney (Wilms'tumor), jiver and adrenal cortex moved us to feview this condition. We teport 14 patients with congenital hemihypertrophy studied from 1974 to 1984 in the Pediatric Surgery and Orthopaedics Departments of Dr. Exequiel González Cortés Hospital.

(Key words: Hemihy pertrophy. Congenital body asymetry).
\end{abstract}

La hemihipertrofia es una de las mas raras e interesantes anomalias del desarrotlo encontradas en la especie humana. Escultores y pintores la exponen en sus obras en la época greco-romana: sin embargo, los primeros casos documentados cientificamente, corresponden al siglo pasado: Meckel 1822 y Wagner $18399-12 \cdot 20-21$.

Hasta el momento, se han encontrado más de 200 comunicaciones en la literatura ${ }^{9}$.

Se define la hemihipertrofia como el sobrecreciniento de uno de los hemicuerpos. completo o parcial, en ausencia de enfermedad vascular, linfática, neurológica o endocrina, manifiesta a la simple inspección y no a la búsqueda acuciosa, ya que comunmente se accpta que individuos normales presenten leves asimetrías entre órganos o segmentos corporales pares ${ }^{6-12}$. Esta asimetría puede ser evidente, en el momento de nacer o hacerse notoria con el transcurso de los años. Una vez desar rollada, parece constante $e^{20}$.

Además del aspecto estético, is importante conocer esta entidad por su asociación con defectos congénitos variađos, especialmente retardo mental y anomalías génito - urinatias ${ }^{2} \cdot 7 \cdot 9 \cdot 17$ - 18-20-21-23. Más aún. la aumentada susceptibilidad de estos niños paral presentar neoplasias de riñón, higado y corteza suprarrendall $12 \cdot 4-5 \cdot 7 \cdot 9-10 \cdot 15-17 \cdot 19 \cdot 20-21 \cdot$ 23 , hace imprescindible un control clínico acucioso y periódico.

Se presentan algunos casos de niños con he-

1 Servicios de Cirugía Pediatricu, Ortopedia y Traumatologia, Hospital Dr. Exequiel González Cortéx.

2 Departamento de Pediatría y Cirugía Infantil Sur, Facultad de Medicina, Universidad de Chile, Santiago. mihipertrofia congénita, detectados en el transcurso de los últimos diez años (1974-1984) y se revisan aspectos de esta afección, básicos para su diagróstico y manejo.

\section{MATERLAL}

Se presentan 14 nirios, de 5 meses a 14 años de edad en el momento del diagnóstico, estudiados y controlados en la policlínica de Cirugía, Ortopedia y Traumatología del Hospital Dr. Exequiel Gonzále? Cortés, desde enero de 1974 a junio de 1984.

La descripción de estos niños se analiza en la Tabla 1.

En las figuras 1 a 6 se muestran algunas de las caracteristicas más relevantes del examen físico y radiológico de esta afección.

En los 14 niños, la hemihipertrofia ha sido asintomática. La mayor parte de los casos consultó por la asimetría corporal, detectada por los padres o por el pediatra en un examen de rutina. Entre las anomalías asociadas, en sólo dos pacientes, se encontró criptorquidea unilateral, que no correspondía, en ninguno de los casos, al lado afectado por el sobrecrecimiento y que fue tratada quirürgicamente.

Una vez establecido el diagróstico de hemjhipertrofia pura, se ha practicado en estos niños, un control riguroso y periódico, no necesitándose hasta el momento de exámenes más especializados. Los estudios neurológicos y psicológicos, revelan normalidad y el desarrollo pondo-estatural ha sido adecuado, comprobándose que la hemihipertrofia se mantiene con el transcurso de los años.

En los casos en que el sobrecrecimiento afecta 
una extremidad inferior, sólo se ha requerido un realce ortopédico contralateral, en el calzado, para evitar así, alteraciones estáticas de la colurn na vertebral.

\section{COMENTARIO}

La verdadera incidencia de la hemihupertrofia congénita, es difícil de determinar, ya que grados leves de asimetría corporal son considerados como normales. Parker ${ }^{17}$ encontró asimetría importante en 10 de 86.000 admisiones de un hospital general de New York. En los archivos de malformaciones congénitas de Birmingham, Inglaterra ${ }^{2}$, aparece una incidencia de 1 por 14.300 recién nacidos vivos. Aparentemente en la afección, no existen factores predisponentes de sexo o raza.

Tabta I.

Lbicación de la hemihipertrofia en 14 pacientes

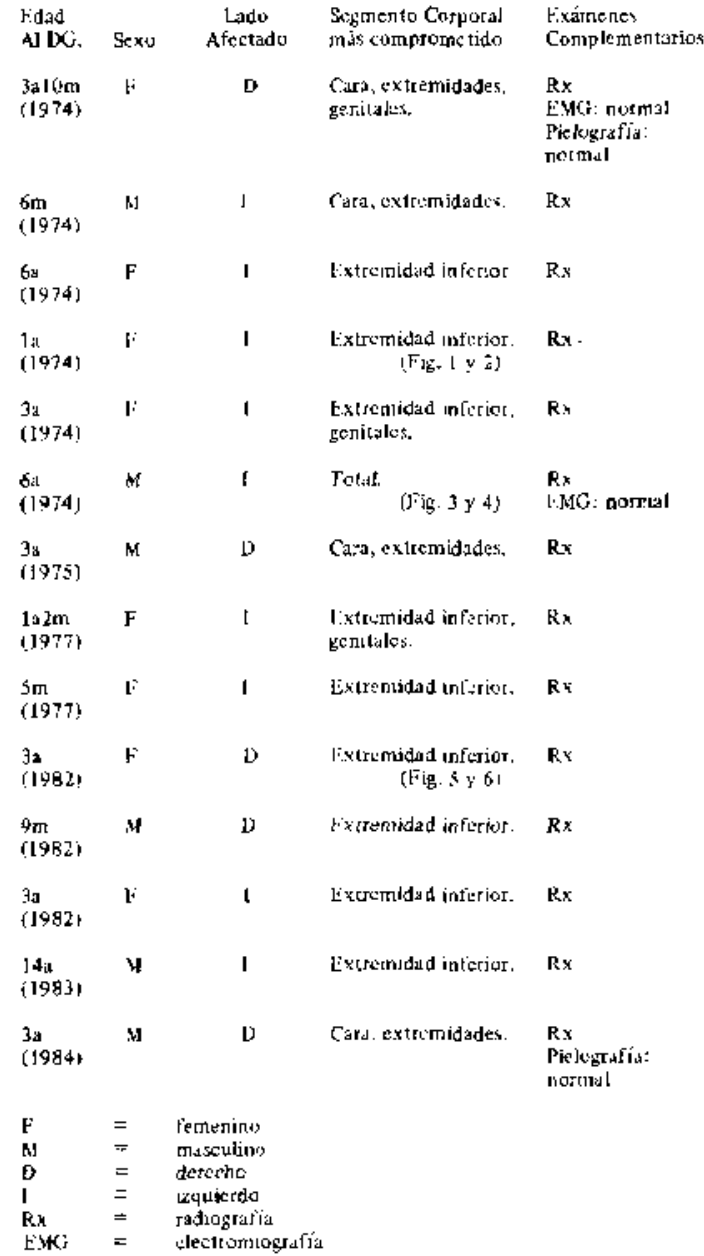

Clínicamente, el sobrecrecimiento puede ser total o predominar en un segmento del hemicuerpo afectado. Más rara es la hemilipertrofia cruzada, que afecta segmentos alternos ${ }^{6}$. Los casos clínicos clásicos, presentan un sobrecrecimiento de un lado del cuerpo, que incluye esqueleto y partes blandas. Ocasionalmente, la piel del lado afectado es más gruesi y el cuero cabelludo es más piloso6 - $9-18-20$. Aproximadamente, $30 \%$ de los casos reportados presentan lesiones del tipo hamartomas, ej. nevus pigmentados, hemangiomas 2-16-18.

El diagnóstico diferencial más importante es con las hemiatrofias de origen neurológico: diastematomielia (lesión medular congénita), hemiplejias congénitas, secuejas de polio, en las cuales, la anamnesis y el examen neurológico, permiten diferenciarlas.

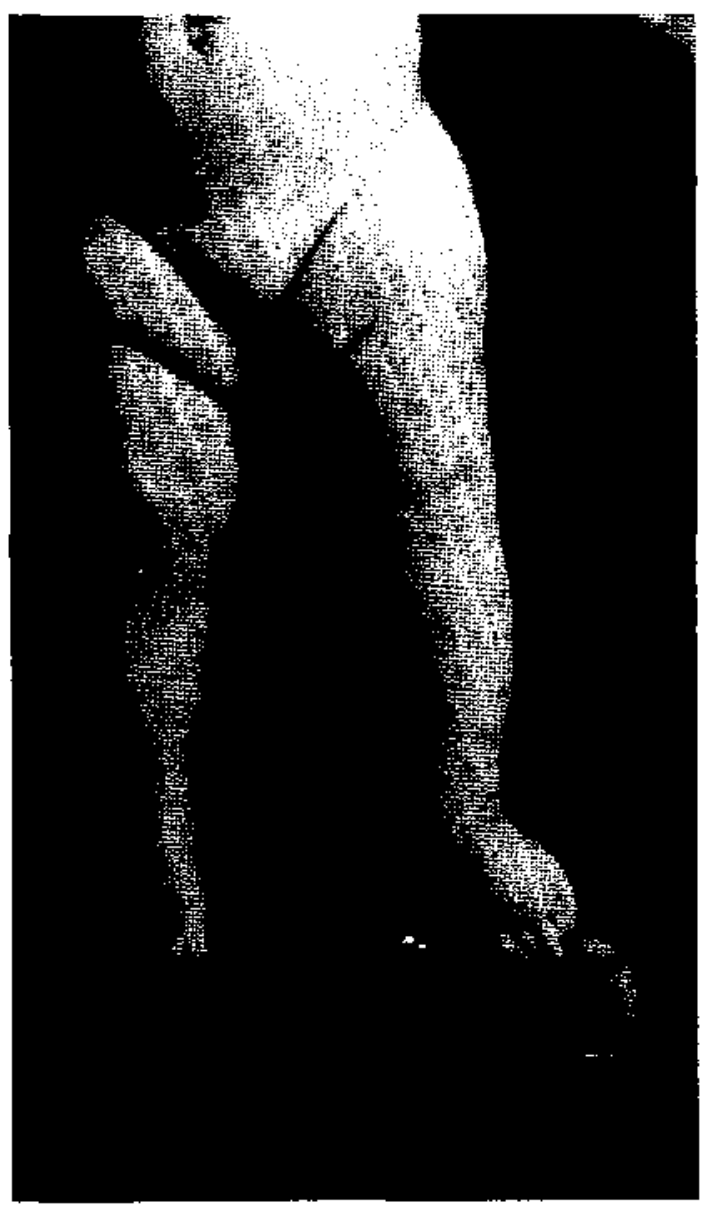

Figura 1.

Hemihipertrofia extremidad inferior jzyuierda. 


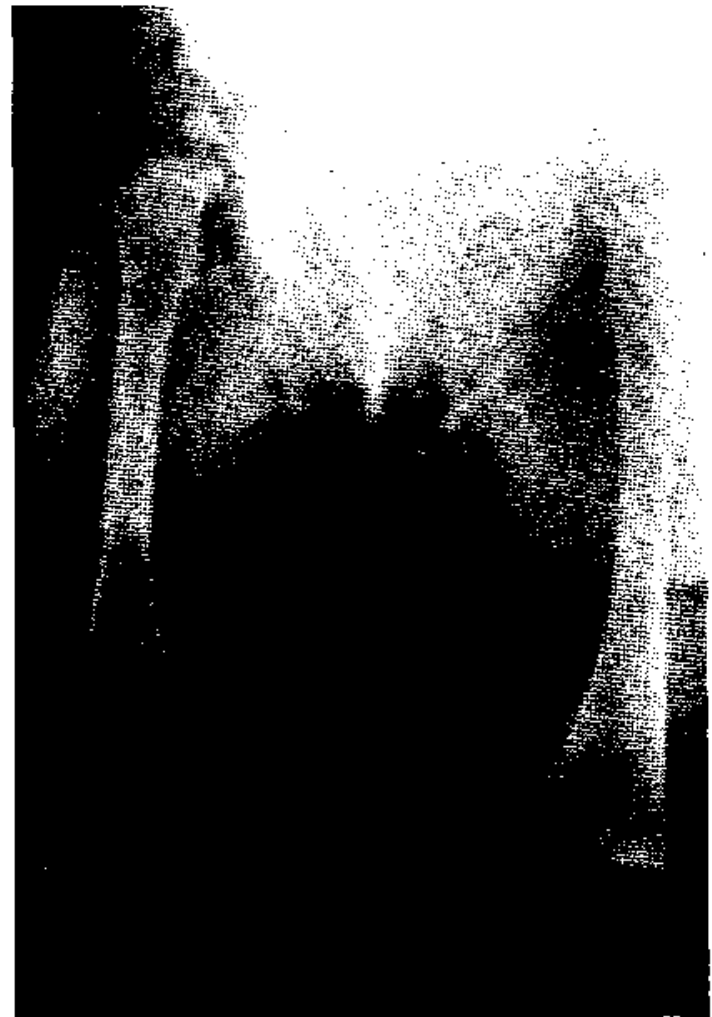

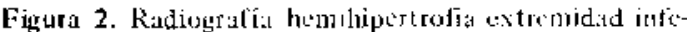
rot izcujerdia.

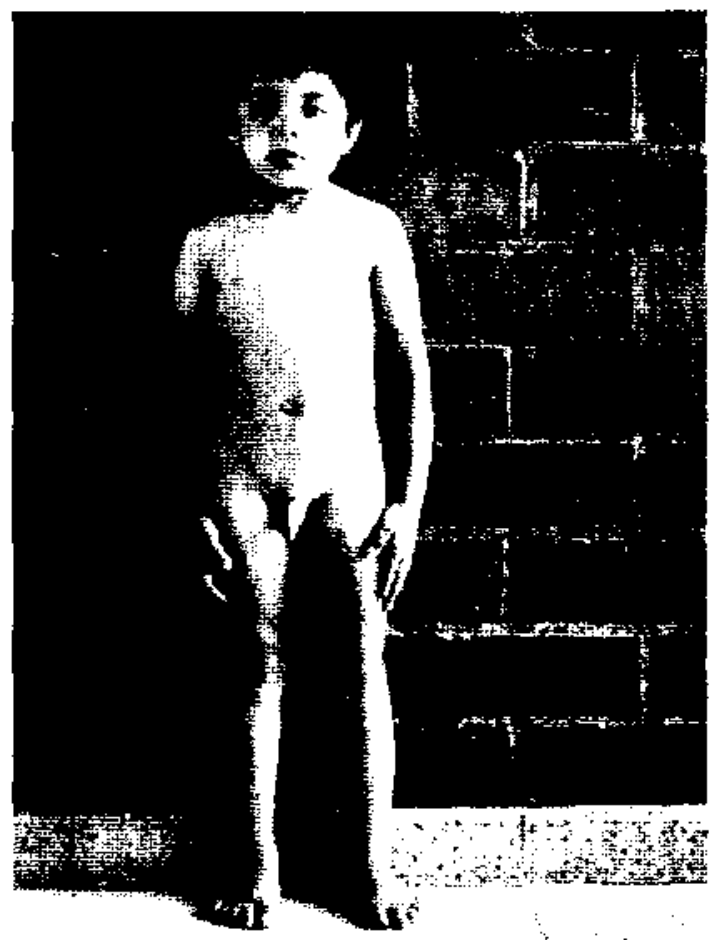

Figura 3. Hemlupertrofia izquierda total.

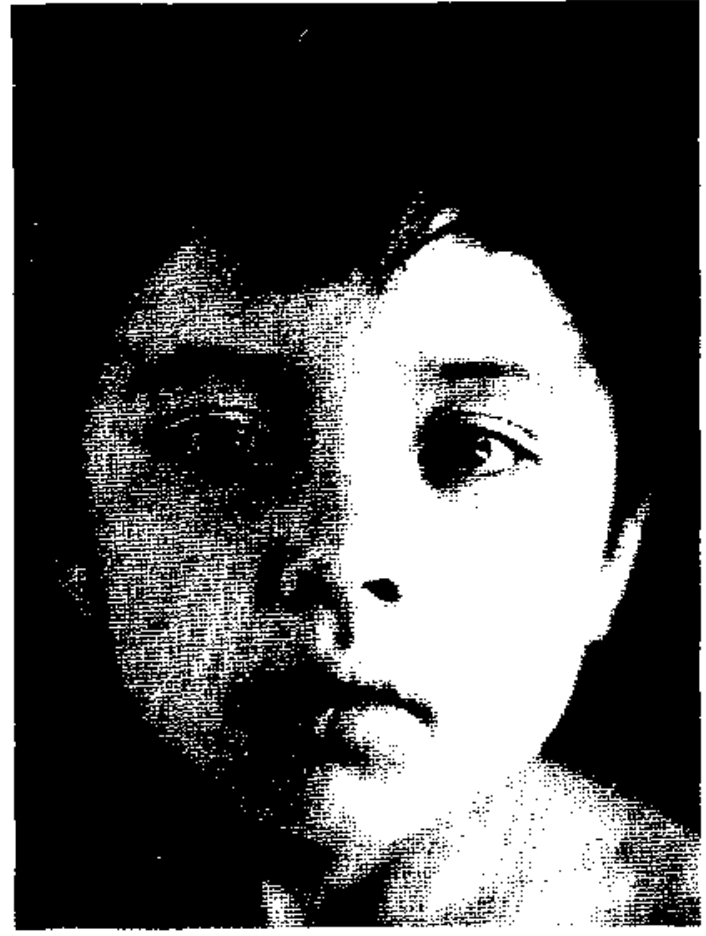

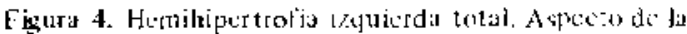
intil.

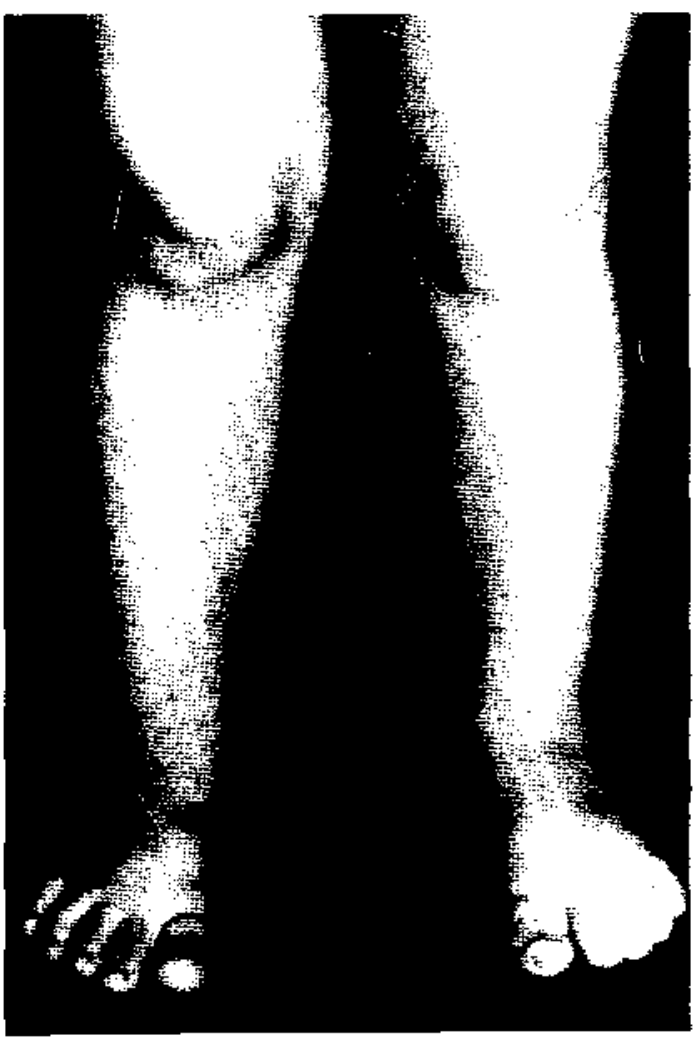

Figura 5. Henihipertrofia extremidad inferior dercectia. 


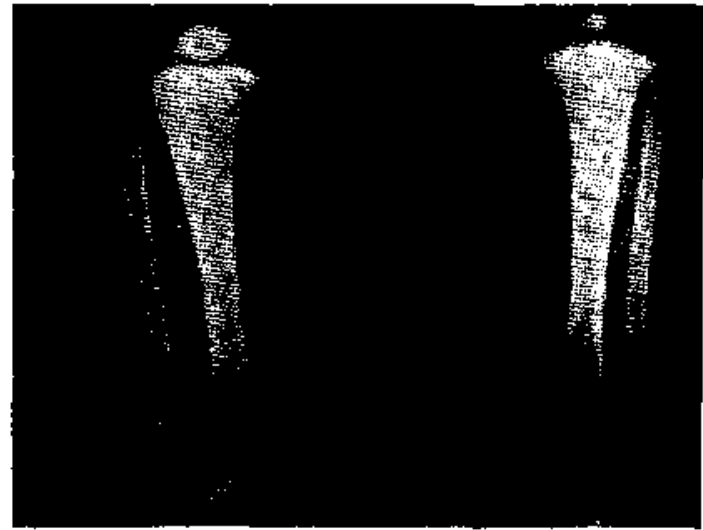

Figura 6. Radiografín del tasto de la tipuras

Otros cuadros que producen una hemihipertrofia corporal real, se observan en la Tabla 2.

Tabla 2.

Hemshipert rofats adquiridas (Secundarials)

I. Causas vasculares o lintáticas

Sindome de Klippe! Tretatuay o neyas warions osteohipertrofico.

Sintrome de Parkes weher o hipertrofie edongec tásica.

Infcrmadad de hilroy o tinfedema compénito.

Fistula arterio venosa congénita

2. Causis neurológicas

Enfermedad de Recklinghausen o netorotibromatosis difusa.

3. Causas endoctinan

Sindrome de Silver

Simdrome de Bfekwth Wiedeman.

Hiperpituisarismo ogtgantismo.

4. Otras

Osteomielitis comiso,

Secuetos de fracturas ate estimukn ef creciminnto.

Dentro de las alasificaciones de la hemihipertrolia corporal encontradis en la literatura, uma de las yue parece más prictica, corresponde a la de Ward y Lerner 2-13.20, que la clasifican en congeritas y adquiridas $y$ ambas en Total (de todos los sistemasi) o Limitada (sólo algunos sistcmas). Las formas tolales putden a sil vez ser fragmenturias, cruzalda o hemilipertrotia $y$ las litnitadas, serlo al sistema muscular, vastular, oseo o neurológico.

La ctiología de la hemihipertretia congénita. aún es desconocida. En 1921. Gesell. platiteo lat prosibilidad de que en estos casos, se prondujera la desviación de un embarazio gemelar, siendo el blastodermo afectado cuantitativamente en una de sus partes ${ }^{12}$. Esto, estaría de acuerdo con la histoputologia de los tejidos de las zonas afecta das, que muestra hiperplasia celular más que una hipertrofia: en estos pacientes ${ }^{2}$. En siete casos se han descritio anomalias cromosomicas. Un paciente tenia un par de cromosomas 16 elongado; sin embargo, no pareciera haber agregación familiar en los casos estudiados, aunque si, un ligero mayor riesgo en los hijos de las madres afectadas-11-15. Tampoco se ha relacionado oon factores ambientales específicos o desusados, por lo the pareciera tener un origen heterogé. neo $2 \cdot 12-15$

Sin considerac la incertidumbre en lo referenti it su etiologia e incidencia, estí clara su asociaéón con una variedad de alteraciones benignas $y$ Iralign as abdotninales y gênito-- urinarias: hipospadia, criptoryudea, ectopia renal, nefromegalis, hipertrolia genital, riñón medular en esponija. El tumor de Wilms es la neoplasia más frecuente, relacionada con la hemihipertrofia y su coexistencia es mayor que la esperada por el azart ${ }^{-2}$. $4 \cdot 5-9 \cdot 10-14-17-20 \cdot 21-23$ Otros tumores con los que también se le reliciona, son el hepatoblastoma y neoplasias adreno corticales:-5-17. Aparentemente se trataría de un sindrome de malformacion--neoplasia (teratogétrico oncogénico) como plantean Knudson y Strong en su hipótesis de lid doble tnuta-

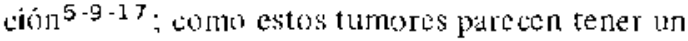
origen embrionario, el cstudio en sut relación con la hemihipertrofia pueds conducir a unia iuformación más especifica de los mecanismos involucrados.

Lo anterior implicit que. annque se trara de una afección rara, un núnero significativo de estos pacientes, licne riesgo de sufrir una neoplitsia abdominal. Por lo tanto, todos los niños con hemilhipertrof ia congénit. deben ser exuminados acuciosamente y seguidos. La cografia abdominal y la pielografïia de elimninuciótl, deben realizarse a la menor sospecina.

Dexde el punto de vista ortopédico, raramente se requiere el empleo de la cirugía. bastando om reitlues ell el calyoulo ${ }^{13}$. Fintre tos tratamientos de frenación oseil, estíln descritas la epifisiódesis de Phemister y la técnica de blount ${ }^{3}-13-19-22$ Estus procedimientus timen indicuciones precisis y deben stectuarse tuando existe diferencia. en las extremidades inferiores, mayor de $4.5 \mathrm{~cm}$. antes de la maduracion osea y mayor a 3,8 cm. despues de ellat $3 \cdot 19$.

\section{CONCLUSION}

Poco se sabe de esta entidad. debido a su aparentemente baja incidencia y el desconociunietrto cie su ctio patngenia. Fl enfoque chinico no solo debe guiarse por las altaraciones estéticas o meénuds. Su relación con patologras benignas 
y malignas abdominales y génito-urinarias, en especial, el tumor de Wilms, exige plantear en estos ninos, un seguimiento clínico periódico, con acucioso examen abdominal, empleando ecografía y pielografía de eliminación a la menor sospecha de masa o proceso citogénico abdominal.

\section{REFERENCIAS}

1 Benson P.F., Vulliamy D.G., Taubman J.O.: Congenital hemihypertrophy and malignancy. The Lancet $1: 468,1963$.

2 Bergsma D.: Birth Defects Compendium. 2a. Ed N.Y. 1979. The National Foundation. March of Dimes. Pág. 458.

${ }^{3}$ Blunt W.P.: Unequal leg length in children. Surg. Clin. of N.A. 38: 10?, 1958.

4 Boxer L.A., Smith D.L.: Wilms'tumor prior to onset of hemihypertrophy. Am. J. Dis. Child. 120:564, 1970 .

\$ Fraumeni J.F., Geiser C.F., Manning M.D.: Wilms'tumor and congenital hemihypertrophy: Report of five new cases and review of literature. Pediatrics 40 : 886, 1967.

${ }^{6}$ Gorlin R.J. Meskin L.H.: Congenital hemihipertrophy. Review of the literature and report of a case with special emphasis on oral manifestations. J. Pedials. 61: 870, 1962.

7 Haicken B.N.: Congenital hemihypertrophy. Problems in long-term management. Am. J. Dis. Child. 120: 372,1970 .

${ }^{8}$ Hammond G.J., Wieben E., Market C.L.: Molecular signals for initiating prolein synthesis in organ hypertrophy. Proc. Natl. Acd. Sci. USA 76: 2455, 1979.

9 Hennessy W.T., Cromie W.J., Duckett J.W.: Congenital hemihypertrophy and associated abdominal lesions. Urology 18: 576,1981 .
${ }^{10}$ Janik J.S., Seeler R.A.: Delayed onset of hemihypertrophy in Wilms'tumor. Case report, 3. Pediats. Surg. 11: 581,1976 .

11 Kääpä $P$., Susiraival P.: Hemihypertrophy with unilateral folliculitis and acne. Case report. Acta Paediatr. Scand. 68: 921, 1979.

12 Mac Ewen G.D., Case J.L.: Congenital hemihypertrophy. A review of 32 cases. Clin. Orthop. 50: 147, 1967.

I ${ }^{3}$ MC Cullogh C.J., Kenwright J.: The prognosis in congenital lower limb hypertrophy. Acta Orthop. Scand. 50: 30?, 1979.

14 Maurer H.S., Pendergrass T.W., Borges W., Honig G.R.: The role of genetic factors in the ctiology of Wilms'tumor. Cancer 43: 205, 1979.

15 Meadows A.T., Lichtenfeld J.L., Koop C.E.: Wilms'tumor in three children of a woman with congenital hemihypertrophy. N. Engl. J, Med. 291: $23,1974$.

16 Muller J.T., Pickett A.B., Frederick F.D.: Facial hemihypertrophy associated with nevus nius lateris syndrome. Oral Surg. 50:226, 1980

17 Parker D.A., Skalko R.G.: Congenital asymmetry: report of 10 cases with assocjated developmental abnormalities. Pediatrics 44: 584, 1969.

18 Pascual Castroviejo 1.: Diagnóstico clínico-radiológico en neurocirugía infantíl. Ed. Científico Mćdica, 1971, pág. 226. Barcelona.

${ }^{19}$ Pedroce A., Coscia P.L., De Guidi G. Le emiipertrofje congenite. Minerva Ortopédica 19: 309, 1968.

20 Ringrose R.E., Jabbour J.T., Keele D.K.: Hemihypertrophie. Review article. Pediatrics 36:434, 1965.

2 I Saypol D.C., laudone V.P.: Congenital hemihypertrophy with adrenal carcinoma and medullary sponge kidney. Urology 21: $510,1983$.

22 Sharrard W,J.W.: Pediatric Orthopaedies and Fractures. Blackwell Scientific Publications, 1971, pag. 427.

${ }^{23}$ Tolchin D., Koenigsberg $M$, Striorineou $M$.: Diagnóstico temprano del tumor de Wilms en una niña can hemihipertrofia $y$ quistes ováricos. Pediatrics (Ed. Esp.) 14:67, 1982 . 